\title{
Management of acute low back pain in the ED: A systematic review.
}

\section{Ashbrook J., Rodgdakis N., Goodwin P., Yeowell G., Callaghan M (2017)}

Emerg Med J. 2017 Dec;34(12): A889. doi: 10.1136/emermed-2017-207308.43.

\section{Abstract}

Study objective There is no consensus on the management of low back pain in the ED and evidence suggests that these patients are likely to receive unwarranted imaging and inappropriate opioid prescription.

The purpose of this study is to review the available literature pertaining to the clinical management of acute low back pain in the ED.

Methods The Preferred Reporting Items for Systematic Reviews and MetaAnalyses (PRISMA) and Realist And Meta-narrative Evidence Syntheses: Evolving Standards (RAMESES) guidelines were observed during this review.

Trials were included if the population studied were adults with acute low back pain in an emergency setting. All diagnostic tests and therapeutic interventions were evaluated.

Methodological quality and risk of bias was appraised using the Downs and Black checklist.

Results 19 articles were identified including 1896 patients that were sub-grouped according to management.

In the pharmacological subgroup corticosteroids were effective in patients with radicular pain, NSAIDs were as effective as other medication with less adverse events, Phenyramidol was not superior to placebo, promethazine and morhpine combined was not more effective than morphine alone and ketamine was no more effective than morphine but had a worse adverse effect profile.

In the emergency transport group TENS and active warming both showed effects in reducing pain, anxiety and heart rate.

In the physical therapy management group less pain and greater satisfaction were reported. 
In the adjunct interventions group showed a trend towards pain reduction in the use of heat/ice packs and short term pain relief in acupuncture and auricular acupuncture.

Conclusions More high quality trials are needed to determine an evidence-based management protocol for the treatment of acute low back pain in the ED. 